\title{
Electrocardiogram Lead
}

National Cancer Institute

\section{Source}

National Cancer Institute. Electrocardiogram Lead. NCI Thesaurus. Code C123976.

An insulated electrical conductor designed to connect to an electrocardiog ram machine. 